\title{
openheart Sex-based outcomes in contemporary antiplatelet therapy trials
}

\author{
Mirvat Alasnag (D) , ${ }^{1}$ Tara L Jones, ${ }^{2}$ Yasmin Hanfi, ${ }^{3}$ Nicola Ryan (D) ${ }^{4}$
}

To cite: Alasnag M, Jones TL, Hanfi Y, et al. Sex-based outcomes in contemporary antiplatelet therapy trials. Open Heart 2021;8:e001761. doi:10.1136/ openhrt-2021-001761

Accepted 13 July 2021

\begin{abstract}
Balancing ischaemic and bleeding risks in highrisk populations undergoing percutaneous coronary interventions has become an everyday dilemma for clinicians. It is particularly difficult to make decisions concerning combinations and duration of antiplatelet regimens in women given the poor representation of women in trials that have shaped current practice. Several contemporary landmark trials have recently been presented at the American College of Cardiology. The trials included the Harmonising Optimal Strategy for Treatment of coronary artery diseases-EXtended Antiplatelet Monotherapy, Ticagrelor With Aspirin or Alone in High-Risk Patients After Coronary Intervention and the TicAgrelor versus CLOpidogrel in Stabilised Patients With Acute Myocardial Infarction. In this article, we summarise the main findings of these trials and include the The Polymer-free Drug-Coated Coronary Stents in Patients at High Bleeding Risk (LEADERS FREE) in search for evidence based best practices for women patients. Although some of these trials had prespecified a subanalysis of sex differences, women constituted only $17 \%-30 \%$ of participants making sex-specific analyses challenging. Data suggest that women benefit from de-escalation to both ticagrelor and clopidogrel monotherapy. However, given the increased bleeding risks observed in women further randomised controlled trials are necessary to determine the most appropriate combination and duration of dual antiplatelet therapy as well as maintenance single antiplatelet therapy.
\end{abstract}

\section{INTRODUCTION}

Current standard antiplatelet therapy after percutaneous coronary intervention (PCI) comprises of dual antiplatelet therapy (DAPT), aspirin plus a P2Y12 inhibitor, for 6-12 months depending on clinical presentation, followed by lifelong single antiplatelet therapy (SAPT), typically low-dose aspirin. ${ }^{12}$ These recommendations are based on trials in which, for the most part, women were underrepresented leading to difficulties with generalisability of the results.

Antiplatelet regimens during and post-PCI involve balancing the risk of ischaemic versus bleeding complications both of which increase morbidity and mortality. ${ }^{3-6}$ Historically, the focus of many trials has been on the reduction of ischaemic risk with bleeding risk playing a secondary role. Several contemporary trials have explored adequately balancing both ischaemic and bleeding risks, with potent antiplatelet agents. STOP-DAPT-2 ${ }^{7}$ and SMART CHOICE, ${ }^{8}$ suggest an abbreviated DAPT regimen that included clopidogrel is noninferior to an extended regimen with respect to bleeding with no increase in ischaemic events. These trials were predominantly in low-risk Asian patients, guided by intracoronary imaging. More importantly, women constituted only $21 \%-27 \%$ of the cohort making it difficult to conduct any sexspecific analyses.

There has been interest in targeting antiplatelet therapy based on platelet reactivity. In the GRAVITAS (Gauging Responsiveness with A VerifyNow assay-Impact on Thrombosis And Safety) study, those with high residual platelet activity after PCI with drugeluting stent (DES) were randomised to standard $(75 \mathrm{mg})$ versus high-dose $(150 \mathrm{mg})$ clopidogrel with no significant differences in the primary ischaemic endpoint or moderate or severe bleeding. ${ }^{9}$ Interestingly, in the double-dose versus standard-dose clopidogrel and high-dose versus low-dose aspirin in individuals undergoing PCI for acute coronary syndromes (CURRENT OASIS 7) study, there was a significant interaction with PCI. Despite no differences in the overall population $^{10}$ in those undergoing PCI the composite primary endpoint of cardiovascular death, myocardial infarction (MI) or stroke at 30 days, was significantly lower in the doubledose clopidogrel cohort. ${ }^{11}$ Although not truly randomised cohorts, as the antiplatelet allocation was determined prior to PCI, there was also a reduction in stent thrombosis (ST) in the double dose clopidogrel group. This reduction in ischaemic risk came at the cost of increased major bleeding.

Various trials including Harmonising Outcomes With Revascularisation and Stents in Acute Myocardial Infarction and Treatment with ADP receptor inhibitorslongitudinal assessment of treatment patterns and events after acute coronary syndrome 

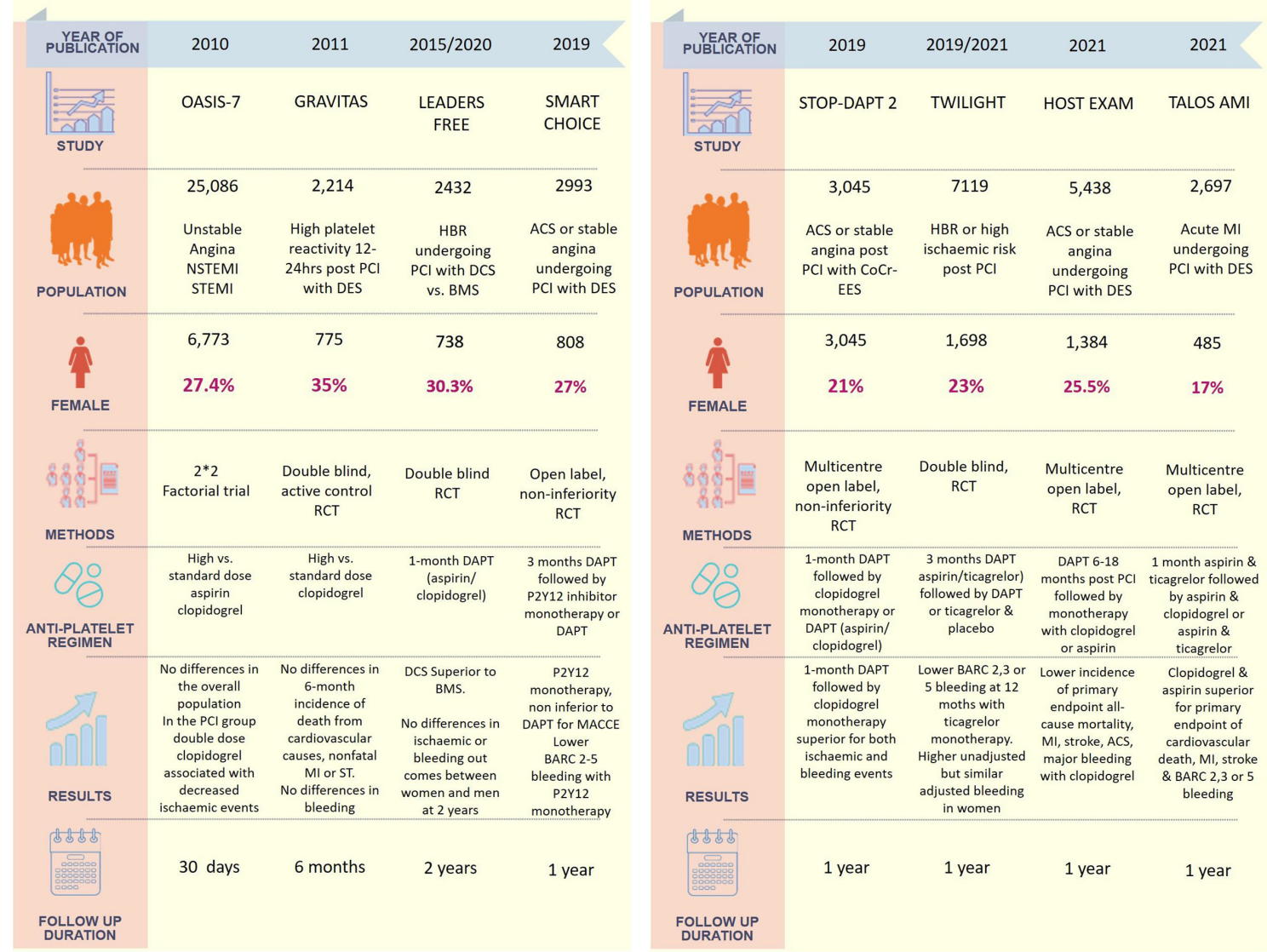

Figure 1 A summary of the most relevant and contemporary antiplatelet trials. AMI, acute myocardial infarction; ACS, acute coronary syndrome; BARC, Bleeding Academic Research Consortium; BMS, bare metal stent; DCS, drug-coated stent; DES, drug-eluting stent; HBR, High Bleeding Risk; HOST EXAM, Harmonising Optimal Strategy for Treatment of coronary artery diseases-EXtended Antiplatelet Monotherapy; MI, myocardial infarction; PCl, percutaneous coronary interventions; RCT, randomised controlled trial; TALOS, TicAgrelor versus CLOpidogrel in Stabilised; TWILIGHT, Ticagrelor With Aspirin or Alone in High-Risk Patients After Coronary Intervention.

have demonstrated that women have a higher bleeding risk than men. ${ }^{12} 13$ The known increased bleeding risk in women as well as conflicting results in clinical trials complicate decision making in women and potentially lead to underutilisation of appropriate percutaneous and medical therapy. ${ }^{1415}$ Furthermore, the optimal DAPT regimen in women, undergoing complex coronary interventions and the optimal chronic SAPT post-PCI remain unclear. In this review, we examine the sex-based analyses of contemporary antiplatelet therapy studies. Figure 1 provides a summary of the design and results of the most relevant and contemporary trials on antiplatelet therapy.

\section{CONTEMPORARY ANTIPLATELET THERAPY TRIALS LEADERS FREE Trial}

The Polymer-free Drug-Coated Coronary Stents in Patients at High Bleeding Risk (LEADERS FREE) trial randomised patients with HBR to PCI with a bare metal stent (BMS) or a polymer-free biolimus A9-eluting drugcoated stent (DCS) with 1 month of DAPT. ${ }^{16}$ A secondary sex-based analysis of the LEADERS FREE aimed to assess 2 years outcomes of HBR patients undergoing PCI. ${ }^{17}$ The protocol mandated 30 days of DAPT with aspirin and a
P2Y 12 inhibitor, preferably clopidogrel, followed by SAPT, preferably with aspirin. Patients in whom a vitamin K antagonist (VKA) was prescribed received either triple therapy or clopidogrel and the VKA for the first 30 days. The primary efficacy endpoint was clinically driven target lesion revascularisation (TLR) and the primary safety endpoint was a composite of cardiac death, MI or ST. Bleeding was assessed using the Bleeding Academic Research Consortium (BARC) scale.

A total of 2432 patients with a mean age of 75 years were included in the trial, similar to most coronary intervention trials, less than a third of participants in this trial were women $30.3 \%$ (738) women and $69.7 \%$ (1694) men. Women were older $(78 \pm 8$ years vs $75 \pm 9$ years, $\mathrm{p}<0.001)$ with a higher prevalence of chronic kidney disease (CKD) $(\mathrm{CrCL}<40 \mathrm{~mL} / \mathrm{min} 24.1 \%$ vs $16.9 \%$, p $<0.001)$. In this study, the characteristics for HBR were different in men and women with women more likely to be considered HBR due to their age, renal function or haemoglobin level, while men were more likely to be considered HBR due to indications for oral anticoagulation $(37.8 \%$ vs $32.4 \%, \mathrm{p}=0.01$ ), planned major surgery within the next 12 months $(17.8 \%$ vs $13.1 \%, p=0.005)$, malignant 
neoplasm $(11.0 \%$ vs $7.0 \%, \mathrm{p}=0.002)$ or hospitalisation for bleeding ( $3.7 \%$ vs $2.2 \%, \mathrm{p}=0.05)$.

The primary efficacy and safety endpoints were similar in men and women with the DCS superior to BMS. Overall, 1 month of DAPT followed by SAPT with aspirin in patients undergoing PCI with appropriate stent platforms appears to be safe with regard to ischaemic outcomes up to 2 years follow-up. Importantly, there were no differences in ischaemic outcomes in women at HBR undergoing PCI compared with men suggesting that clinically indicated PCI should not be withheld from women with HBR. Similarly, TLR was improved with DCS compared with BMS therefore BMS does not appear to have a role in PCI in women where appropriate DCS are available.

With regard to bleeding outcomes, overall there were similar rates of BARC types 3-5 major bleeding at 2 years, however, women experienced more BARC 3-5 bleeding at 30 days compared with men, likely reflecting the lower rates of radial access in women compared with men. In both sexes, BARC types $2-5$ bleeding was lower with radial access compared with femoral. However, even when radial access was used in women they had increased rates of access site bleeding compared with men; radial access in women had similar rates of BARC 2-5 bleeding as femoral access in men. Major bleeding, both vascular and non-vascular, was associated with increased 2-year mortality in both sexes, therefore, bleeding avoidance strategies during PCI are critical in all patients particularly women.

\section{Harmonising Optimal Strategy for Treatment of coronary artery diseases-EXtended Antiplatelet Monotherapy trial}

The Harmonising Optimal Strategy for Treatment of coronary artery diseases-EXtended Antiplatelet Monotherapy study was an investigator-initiated, multicentre, prospective, randomised, open-label trial conducted at 37 sites in South Korea. ${ }^{18}$ The population included patients $>20$ years of age who had been maintained on DAPT with aspirin plus any P2Y12 inhibitor for 6-18 months following PCI with a DES. Patients with ischaemic or bleeding events were excluded from randomisation. Importantly, there were no restrictions based on the index PCI such as diagnosis or indication, lesion location or length or number of lesions or vessels treated. Patients were randomised in a 1:1 fashion to receive either clopidogrel $75 \mathrm{mg}$ daily or aspirin $100 \mathrm{mg}$ daily. Follow-up was at 12 and 24 months postrandomisation. The primary endpoint was a composite of all-cause mortality, non-fatal MI, stroke, readmission for acute coronary syndrome (ACS) and major bleeding (defined as BARC type 3 or greater) within the 24-month follow-up period. Hazards modelling was performed for prespecified subgroup analysis including sex.

A total of 5438 patients with a mean age of 63.5 years were successfully randomised. Of this population, only $25.5 \%(\mathrm{n}=1384)$ were female. The median time from PCI to randomisation was 382 days. During the 24-month follow-up period, the primary endpoint occurred in 152 patients $(5.7 \%)$ in the clopidogrel group versus 207 patients $(7.7 \%)$ in the aspirin group $(\mathrm{p}=0.0035)$, with an absolute risk reduction of $2.0 \%$. This was largely driven by reduction in the thrombotic composite endpoint ( $\mathrm{p}=0.003)$ and major bleeding $(\mathrm{p}=0.035)$ in favour of the clopidogrel group. Interestingly, the incidence of allcause mortality was similar between the two groups $(1.9 \%$ in the clopidogrel group vs $1.3 \%$ in the aspirin group, $\mathrm{p}=0.101$ ).

Regarding the subgroup analysis according to sex, a trend towards benefit with respect to the primary endpoint was seen in the clopidogrel group among female patients though the benefit did not reach statistical significance $(p=0.275)$ due to overall low number of female participants. However, post hoc analysis showed no statistically significant interaction between treatment effect and sex $(p=0.63)$. A similar trend was seen with regards to composite thrombotic events and any bleeding events among females, but again these results did not reach statistical significance $(\mathrm{p}=0.060$ for thrombotic events, $p=0.522$ for major bleeding). While the trend towards benefit of clopidogrel monotherapy over aspirin in women is hypothesis generating, ultimately the overall benefit in women remains unproven. Although sex was a prespecified subgroup analysis, the lack of sex stratification at randomisation and additional details regarding comorbidities and individual clinical details within this group makes interpretation of these results more challenging. Furthermore, patients with prior ischaemic or bleeding events were excluded from this trial.

Of particular, interest is the secondary endpoint of any bleeding, which arguably proved least impressive in the female population. While results trended towards benefit in the clopidogrel monotherapy group, this result did not approach statistical significance $(\mathrm{p}=0.522)$. As demonstrated in prior studies, bleeding among women is of particular concern and more data are needed with regard to this risk in the female patient population. ${ }^{19} 20$ Finally, this study was conducted in South Korea, and the included population was almost exclusively east Asian patients. The lack of diversity among study participants may limit the ability to extrapolate results on a broader scale.

\section{Ticagrelor With Aspirin or Alone in High-Risk Patients After Coronary Intervention Trial}

The Ticagrelor With Aspirin or Alone in High-Risk Patients After Coronary Intervention (TWILIGHT) study was a multicentric investigator-initiated, placebocontrolled randomised trial conducted in 187 sites worldwide ${ }^{21}$ At 3 months post-PCI, patients, at high risk for ischaemia or bleeding, adherent to DAPT with ticagrelor and aspirin and no major adverse events were randomised to ticagrelor plus aspirin or ticagrelor plus placebo for 1 year. After the 12-month period mandated by the study protocol, the antiplatelet was assigned at the discretion of their treating physician with an additional 
3 months of follow-up. The primary endpoint was BARC type 2, 3 or 5 bleeding. The primary ischaemic endpoint was a composite of death, MI (in accordance with the third universal definition) or stroke, revascularisation and ST.

A prespecified secondary analysis assessed outcomes by sex. ${ }^{22}$ A total of 7119 patients with a mean age of 63.9 years were included $23.9 \%$ of women. Women were older $(65.5$ years vs 63.4 years), with a significantly higher prevalence of CKD (21.2\% vs $14.7 \%)$ and anaemia. Fewer women had prior MI, PCI or coronary artery bypass grafting with similar angiographic features including chronic total occlusions or bifurcation stenting. The primary bleeding endpoint occurred more often in women than men, however, after multivariate adjustment, bleeding risk associated with female sex was not significant (adjusted HR, 1.20; 95\% CI) suggesting bleeding risk was attributable to baseline characteristics. The ischaemic endpoints were similar in both sexes.

Although sex was a prespecified subgroup of the TWILIGHT study, the total number of women enrolled was low and randomisation was not stratified by sex. Overall, the early withdrawal of aspirin and ticagrelor monotherapy appears beneficial in women, however, the increased bleeding risk needs further assessment. The population was high risk, therefore, the results cannot be extrapolated to lower risk women with stable disease.

\section{TicAgrelor versus CLOpidogrel in Stabilised Patients With AMI trial}

TicAgrelor versus CLOpidogrel in Stabilised Patients With Acute Myocardial Infarction (TALOS-AMI) a multicentre, randomised, open-label trial of 2697 undergoing PCI for AMI was presented at the American College of Cardiology Virtual Annual Scientific Session, ACC 2021 16 May 2021. All patients received aspirin and ticagrelor for 1-month postsuccessful PCI. Patients with no adverse events at 1 month were randomised to aspirin $100 \mathrm{mg}$ plus clopidogrel $75 \mathrm{mg}$ daily (de-escalation, $\mathrm{n}=1349$ ) or a multicentre, randomised, open-label trial of 2697 undergoing PCI for AMI aspirin $100 \mathrm{mg}$ plus ticagrelor $90 \mathrm{mg}$ two times daily. The primary endpoint was a composite of cardiovascular death, MI, stroke, and BARC bleeding type 2, 3 or 5 from 1 to 12 months after the index PCI.

Overall, the primary endpoint occurred in $4.6 \%$ of the de-escalation arm and $8.2 \%$ of the Aspirin plus Ticagrelor arm $(\mathrm{p}<0.001$ for both noninferiority and superiority) driven by a reduction in major bleeding with a numerically lower rate of ischaemic endpoints with de-escalation therapy. Overall, only $17 \%$ of the participants were female and given that the paper has not been published it is difficult to determine sex-based differences in de-escalation. TALOS AMI mirrors the results of the European single-centre TOPIC $^{23}$ trial, which again showed reduced bleeding complications in the de-escalation arm, however, included $<20 \%$ women.

\section{CONCLUSION}

Modern PCI DAPT regimens are complex requiring adequate balancing of both ischaemic and bleeding risks, several contemporary trials have addressed the bleeding and ischaemic risks of varying combinations and durations of potent antiplatelet agents. Despite the prespecified substudies in several trials, women constituted $17 \%-30 \%$ of participants making sex-specific analyses challenging. From the data available, it would appear that women benefit from de-escalation to both ticagrelor and clopidogrel monotherapy. Given the increased bleeding risks observed in women, further randomised controlled trials determining the most appropriate combination and duration of DAPT as well as maintenance SAPT are required.

Twitter Mirvat Alasnag @mirvatalasnag, Tara L Jones @taraloujones, Yasmin Hanfi @Jazzminem2008 and Nicola Ryan @NicolaR36291888

Contributors All authors contributed equally to all parts of this article.

Funding The authors have not declared a specific grant for this research from any funding agency in the public, commercial or not-for-profit sectors.

Competing interests None declared.

Patient consent for publication Not required.

Provenance and peer review Not commissioned; internally peer reviewed.

Open access This is an open access article distributed in accordance with the Creative Commons Attribution Non Commercial (CC BY-NC 4.0) license, which permits others to distribute, remix, adapt, build upon this work non-commercially, and license their derivative works on different terms, provided the original work is properly cited, appropriate credit is given, any changes made indicated, and the use is non-commercial. See: http://creativecommons.org/licenses/by-nc/4.0/.

ORCID iDs

Mirvat Alasnag http://orcid.org/0000-0002-8714-0334

Nicola Ryan http://orcid.org/0000-0002-2451-8790

\section{REFERENCES}

1 Neumann F-J, Sousa-Uva M, Ahlsson A. ESC/EACTS guidelines on myocardial revascularization. Eur Heart J 2018 https://academic. oup.com/eurheartj/advance-article/doi/10.1093/eurheartj/ehy394/ 5079120

22016 ACC/AHA guideline focused update on duration of dual antiplatelet therapy in patients with coronary artery disease: a report of the American College of Cardiology/American heart association Task force on clinical practice guidelines. J Am Coll Cardiol.

3 Chhatriwalla AK, Amin AP, Kennedy KF, et al. Association between bleeding events and in-hospital mortality after percutaneous coronary intervention. JAMA 2013;309:1022.

4 Mehran R, Pocock S, Nikolsky E, et al. Impact of bleeding on mortality after percutaneous coronary intervention. JACC Cardiovasc Interv 2011;4:654-64.

5 Jernberg T, Hasvold P, Henriksson M, et al. Cardiovascular risk in post-myocardial infarction patients: nationwide real world data demonstrate the importance of a long-term perspective. Eur Heart $J$ 2015;36:1163-70.

6 Kwok CS, Rao SV, Myint PK, et al. Major bleeding after percutaneous coronary intervention and risk of subsequent mortality: a systematic review and meta-analysis. Open Heart 2014;1:e000021.

7 Watanabe H, Domei T, Morimoto T, et al. Effect of 1-Month dual antiplatelet therapy followed by clopidogrel vs 12-month dual antiplatelet therapy on cardiovascular and bleeding events in patients receiving $\mathrm{PCl}$ : the STOPDAPT-2 randomized clinical trial. JAMA 2019;321:2414.

8 Hahn J-Y, Song YB, Oh J-H, et al. Effect of P2Y12 inhibitor monotherapy vs dual antiplatelet therapy on cardiovascular events in patients undergoing percutaneous coronary intervention: the SMART-CHOICE randomized clinical trial. JAMA 2019;321:2428. 
9 Price MJ, Berger PB, Teirstein PS, et al. Standard- vs high-dose clopidogrel based on platelet function testing after percutaneous coronary intervention: the GRAVITAS randomized trial. JAMA 2011;305:1097.

10 Dose comparisons of clopidogrel and aspirin in acute coronary syndromes. N Engl J Med Overseas Ed 2010;363:930-42.

11 Mehta SR, Tanguay J-F, Eikelboom JW, et al. Double-dose versus standard-dose clopidogrel and high-dose versus low-dose aspirin in individuals undergoing percutaneous coronary intervention for acute coronary syndromes (CURRENT-OASIS 7): a randomised factorial trial. Lancet 2010;376:1233-43.

$12 \mathrm{Yu}$ J, Mehran R, Grinfeld L, et al. Sex-based differences in bleeding and long term adverse events after percutaneous coronary intervention for acute myocardial infarction: three year results from the HORIZONS-AMI trial. Catheter Cardiovasc Interv 2015;85:359-68.

13 Hess CN, McCoy LA, Duggirala HJ, et al. Sex-Based differences in outcomes after percutaneous coronary intervention for acute myocardial infarction: a report from TRANSLATE-ACS. J Am Heart Assoc 2014;3 https://www.ahajournals.org/doi/

14 Mehta LS, Beckie TM, DeVon HA, et al. Acute myocardial infarction in women. Circulation 2016;133:916-47.

15 Chandrasekhar J, Baber U, Sartori S, et al. Sex-Related differences in outcomes among men and women under 55 years of age with acute coronary syndrome undergoing percutaneous coronary intervention: results from the prometheus study. Catheter Cardiovasc Interv 2017;89:629-37.

16 Urban P, Meredith IT, Abizaid A, et al. Polymer-Free Drug-Coated coronary stents in patients at high bleeding risk. N Engl J Med 2015;373:2038-47.
17 Mehran R, Chandrasekhar J, Urban P, et al. Sex-Based outcomes in patients with a high bleeding risk after percutaneous coronary intervention and 1-Month dual antiplatelet therapy: a secondary analysis of the leaders free randomized clinical trial. JAMA Cardiol 2020;5:939-47.

18 Koo B-K, Kang J, Park KW, et al. Aspirin versus clopidogrel for chronic maintenance monotherapy after percutaneous coronary intervention (HOST-EXAM): an investigator-initiated, prospective, randomised, open-label, multicentre trial. Lancet 2021;397:2487-96.

19 Chichareon P, Modolo R, Kerkmeijer L, et al. Association of sex with outcomes in patients undergoing percutaneous coronary intervention: a subgroup analysis of the global leaders randomized clinical trial. JAMA Cardiol 2020;5:21-9.

20 Mrdovic I, Savic L, Asanin M, et al. Sex-related analysis of short- and long-term clinical outcomes and bleeding among patients treated with primary percutaneous coronary intervention: an evaluation of the RISK-PCl data. Can J Cardiol 2013;29:1097-103.

21 Mehran R, Baber U, Sharma SK, et al. Ticagrelor with or without aspirin in high-risk patients after PCl. N Engl J Med 2019;381:2032-42.

22 Vogel B, Baber U, Cohen DJ, et al. Sex differences among patients with high risk receiving ticagrelor with or without aspirin after percutaneous coronary intervention: a subgroup analysis of the twilight randomized clinical trial. JAMA Cardiol 2021. doi:10.1001/ jamacardio.2021.1720. [Epub ahead of print: 15 May 2021].

23 Cuisset T, Deharo P, Quilici J, et al. Benefit of switching dual antiplatelet therapy after acute coronary syndrome: the topic (timing of platelet inhibition after acute coronary syndrome) randomized study. Eur Heart J 2017;38:3070-8. 